\title{
Magnetic Retraction of Bowel by Intraluminal Injectable Cyanoacrylate-Based Magnetic Glue
}

\author{
Zhigang Wang, ${ }^{1}$ Andrew Brown,, Pascal André, ${ }^{1}$ Stuart I. Brown, \\ Gordon J. Florence, ${ }^{3}$ and Alfred Cuschieri ${ }^{1}$ \\ ${ }^{1}$ Institute for Medical Science and Technology (IMSaT), Wilson House, University of Dundee, Dundee DD2 1FD, UK \\ ${ }^{2}$ School of Physics and Astronomy, University of St Andrews, St Andrews KY16 9SS, UK \\ ${ }^{3}$ School of Chemistry, University of St Andrews, St Andrews KY16 9ST, UK
}

Correspondence should be addressed to Alfred Cuschieri; a.cuschieri@dundee.ac.uk

Received 14 August 2013; Accepted 1 October 2013

Academic Editor: Elena Landi

Copyright (C) 2013 Zhigang Wang et al. This is an open access article distributed under the Creative Commons Attribution License, which permits unrestricted use, distribution, and reproduction in any medium, provided the original work is properly cited.

\begin{abstract}
Magnetic retraction offers advantages over physical retraction by graspers because of reduced tissue trauma. The objectives of this study are to investigate a novel method of magnetisation of bowel segments by intraluminal injection of magnetic glue and to demonstrate the feasibility of magnetic retraction of bowel with sufficient force during minimal access surgery. Following an initial materials characterisation study, selected microparticles of stainless steel (SS410- $\mu$ Ps) were mixed with chosen cyanoacrylate glue (Loctite 4014). During intraluminal injection of the magnetic glue using ex vivo porcine colonic segments, a magnetic probe placed at the injected site ensured that the SS410- $\mu$ Ps aggregated during glue polymerisation to form an intraluminal mucosally adherent coagulum. The magnetised colonic segments were retracted by magnetic probes $(5$ and $10 \mathrm{~mm})$ placed external to the bowel wall. A tensiometer was used to record the retraction force. With an injected volume of $2 \mathrm{~mL}$ in a particle concentration of $1 \mathrm{~g} / \mathrm{mL}$, this technique produced maximal magnetic retraction forces of $2.24 \pm 0.23 \mathrm{~N}$ and $5.11 \pm 0.34 \mathrm{~N}(n=20)$, with use of 5 and $10 \mathrm{~mm}$ probes, respectively. The results indicate that the formation of an intraluminal coagulum based on SS410- $\mu$ Ps and Loctite 4014 produces sufficient magnetic retraction for bowel retraction.
\end{abstract}

\section{Introduction}

Retraction of bowel loops during minimal access surgery (MAS) remains problematic because the low friction slippery smooth and moist surface resists grasping. For this reason, to provide effective traction, the jaws of laparoscopic graspers feature ridges or toothed profiles to minimize slip and for this reason, the bowel is often grasped too tightly, increasing risk of trauma and associated complications, for example, delayed healing, adhesion formation, and even perforation [1]. The potential bowel trauma during laparoscopic grasping is well documented. One study [2] confirmed the low incidence of successful grasping (62\%) and highlighted the need for improvements in laparoscopic grasping. This is particularly pertinent to natural-orifice transluminal endoscopic surgery (NOTES) and single port or port laparoscopic surgery (SPLS). Thus exposure of the gallbladder [3] is difficult during SPL cholecystectomy. Ryou and Thompson [4] described the use of internal and external magnets for liver retraction during experimental transcolonic (NOTES) cholecystectomy and found that the magnetic system provided effective liver retraction and significantly shortened the procedure time.

Magnetic interactions and magnetic force have attracted considerable research for both medical and surgical applications $[5,6]$. Magnetic microparticles and small magnets have been used for MAS applications such as in magnetic tissue retraction $[4,7,8]$, magnetized islets separation for transplantation [9], magnetic navigation of catheters [10] or untethered devices (e.g., microrobots or magnetic capsules) [11-13], magnetic detection and marker [14-16], and magnetic compression anastomosis $[17,18]$. Magnetic fields generated by magnetic particles [14] or implanted magnets [16] have been used for the detection of tumor or lesion sites. Clinically the strong magnetic interaction between paired magnets has been used to create a compression anastomosis for revision of bilioenteric anastomotic stricture in a patient 
after live-related hepatic transplantation [17] and to create a choledochojejunal anastomosis [18].

We have been investigating tissue magnetization by magnetic nano/microparticles for MAS applications and have previously reported two tissue ferromagnetisation approaches for retraction: (i) surface magnetization by applying a small volume of glue-based magnetic media to the mucosal surface [7] and (ii) by interstitial injection of phosphate-buffered saline (PBS) ferrofluids [8]. In these experiments injected ferromagnetisation was shown to be superior to magnetisation by surface magnetic pellets as the latter tended to peel off the tissue during retraction by magnetic probes.

In the present study, we report a third novel approach for the magnetization of bowel loops for magnetic retraction during MAS and open surgery. This is based on intraluminal injection of glue-based magnetic glue which bonds on polymerization to the mucosal layer of the bowel wall. A magnetic probe is then inserted intraperitoneally and placed on the serosal aspect to retract the magnetized bowel. This paper reports the development and characterization of cyanoacrylate magnetic glues containing dispersed stainless steel microparticles.

\section{Methods}

2.1. Glues and Maximal Temperatures during Polymerisation. Four medical grade glues were investigated for their suitability in the formulation of injectable magnetic glues: Dermabond (Ethicon, Somerville, NJ, USA), Indermil (US Surgical, Norwalk, NJ, USA), and Histoacryl (Braun, Melsungen, Germany) and Loctite 4014 (Henkel, Dusseldorf, Germany). All are topical skin adhesives made of octyl-cyanoacrylate or butyl-cyanoacrylate glues [19]. Since temperatures of $42^{\circ} \mathrm{C}$ and above are known to damage tissues [20], the extent of maximal heat generation during glue polymerisation was investigated. The heat sink effect resulting from perfusion of the bowel wall was simulated by circulation of isotonic solution by means of a pump on the serosal surface of the colon segments which were placed in a water bath. Figure 1 illustrates a thermal camera (Cedip Jade camera, FLIR Systems, France) temperature measurement system in an open bowel experiment within a humidity chamber. In this initial study $0.5 \mathrm{~mL}$ of glue was deposited onto the surface of the bowel segment which was kept moist at $37^{\circ} \mathrm{C}$ with Hartmann's solution circulating at $7 \mathrm{~L} / \mathrm{min}$ to simulate in vivo conditions including the heat sink of vascular perfusion.

\subsection{Characterization of Magnetic Microparticles. Two types} of stainless steel microparticles (SS- $\mu \mathrm{Ps}$ ) were examined in the initial materials characterisation: 410 microparticles (SS410- $\mu$ Ps, Goodfellow Cambridge Ltd., Huntingdon, UK) and 430 microparticles (SS430- $\mu$ Ps, from Alfa Aesar, A Johnson Matthey Company, Lancashire, UK). Both materials had been previously reported in the literature $[15,21,22]$ for use in medical/surgical applications. The magnetic properties of the two materials were quantified with a Superconducting Quantum Interference Device (SQUID) magnetometer

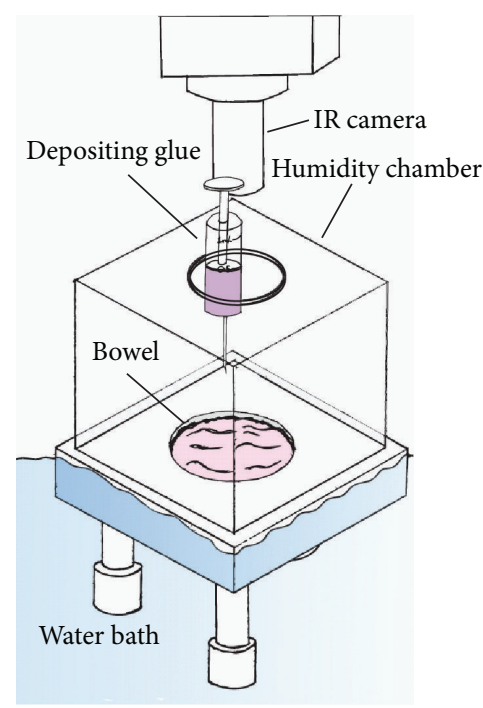

FIGURE 1: Setup for study of heat generation during polymerisation of the glues in an open bowel segment within a closed chamber.

(MPMS XL from Quantum Design, now part of Lot-Oriel $\mathrm{GmbH} \& \mathrm{Co}$. KG). The particle crystallinity was confirmed by X-ray diffraction (XRD; STADI/P powder diffractometer from Stoe) and their sizes were determined by scanning electron microscopy (SEM; S-4800 from Hitachi). Chemical composition of all particles was also analysed by inductively coupled plasma optical emission spectrometry (ICP-OES; Optima 5300 DV from Perkin Elmer).

2.3. Cyanoacrylate-Based Magnetic Glue. Loctite 4014 was chosen to formulate the injectable cyanoacrylate-based magnetic glue in view of its lower cost ease of use (single component) and its quick cure rate in high humidity conditions. Due to its very low viscosity, a range of concentrations can be mixed with magnetic particles for different applications. Concentrations of particles ranging from 0.5 to $1 \mathrm{~g} / \mathrm{mL}$ were used for the magnetic bowel retraction studies. In the formulation of high concentration magnetic glue $(1 \mathrm{~g} / \mathrm{mL}$ magnetic fluid), 2.0 grams of SS410 magnetic particles was placed in a Sure/Seal bottle and $2 \mathrm{~mL}$ Loctite 4014 was added thereafter. The magnetic particles were then mixed and suspended in the liquid glue by vigorous hand shaking of the sealed bottle.

2.4. Magnetic Bowel Retraction. Figures 2(a) and 2(b) illustrates the components of the intraluminal retraction system for MAS procedure: (i) specially designed intra-abdominal injection probe was used with suction openings at its end to facilitate intraluminal injection of magnetic glue media into a bowel segment (Figure 2(a)); (ii) magnetic probe is held on the serosal side adjacent to the site of injection to ensure formation of a strongly magnetic coagulum, which adheres to the mucosal surface on polymerisation (Figure 2(b)); (iii) the magnetised bowel can then be retracted by the external magnetic probe.

We used neodymium iron boron $(\mathrm{NdFeB})$ disc magnets with a remanence of $1.20 \mathrm{~T}$ (grade N35, Eclipse 


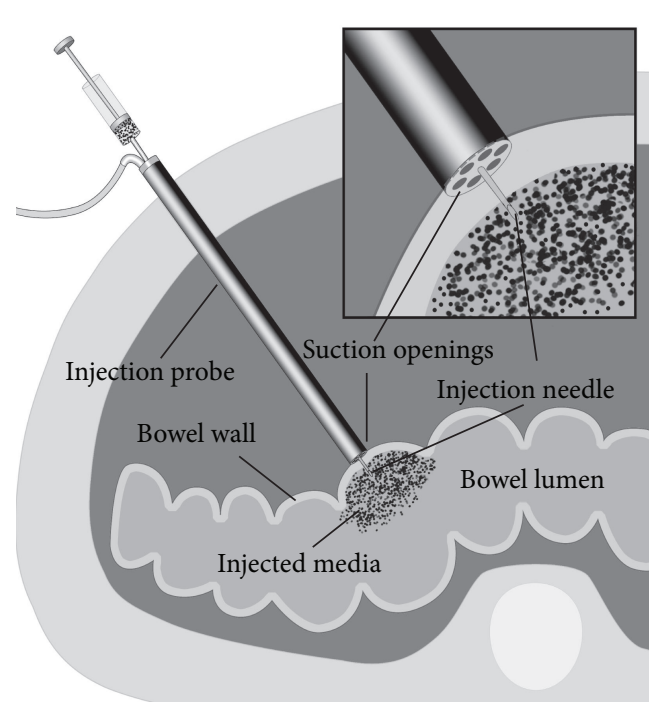

(a)

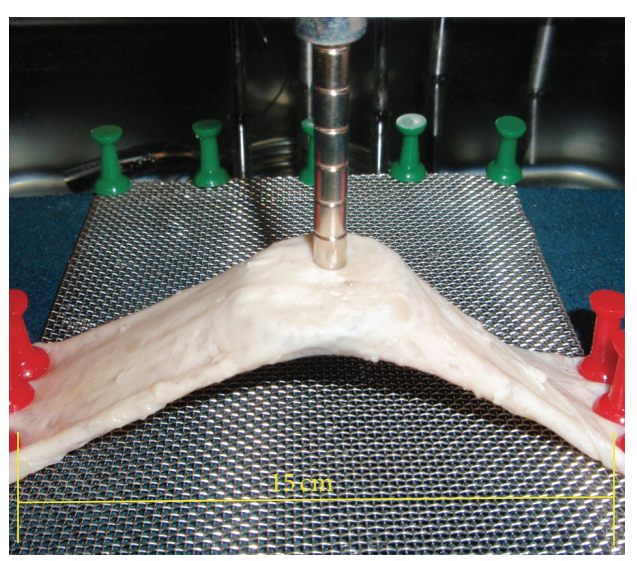

(c)

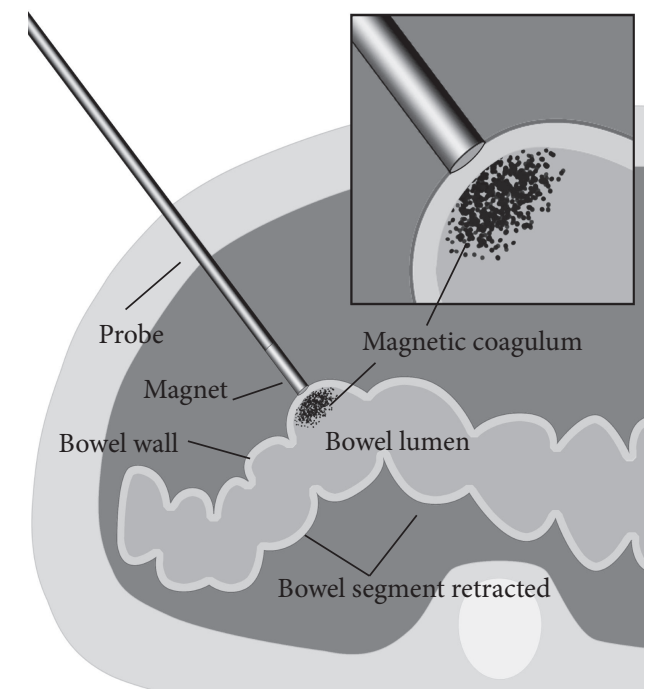

(b)

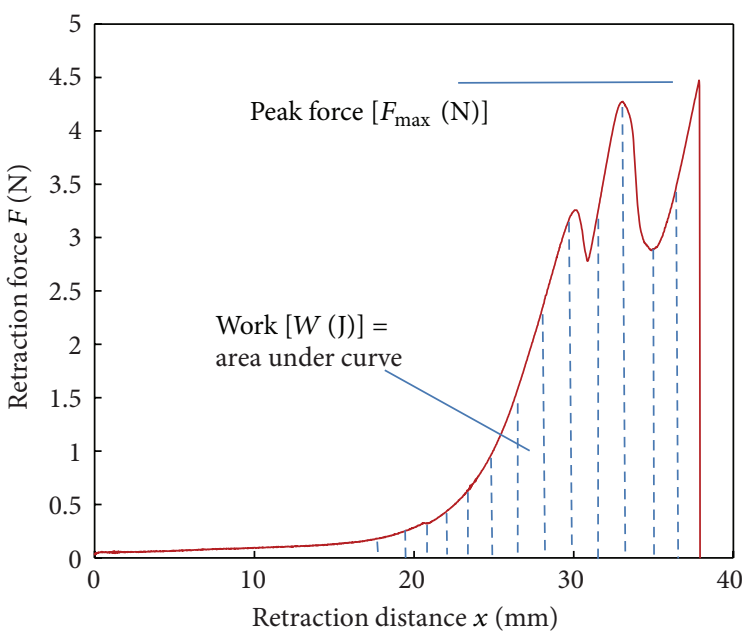

(d)

FIGURE 2: Magnetic retraction by intraluminal injection bowel magnetization. (a) Illustration of an intra-abdominal injection probe with suction openings for capturing bowel wall to facilitate intraluminal injection. (b) Illustration of an intra-abdominal magnet probe with distal magnet interacting with the injected magnetic glue attracted to the inner wall of the lumen by the abdominal magnet probe. (c) Photograph of an ex vivo porcine bowel experimental setup for magnetic bowel retraction force measurement. (d) Illustration of a recorded retraction force-distance curve for calculating peak force and work.

Magnetics Ltd., Sheffield, UK) of two diameters: $5 \mathrm{~mm}$ and $10 \mathrm{~mm}$. Increasing the magnet's axial length can increase its magnetic field strength and attraction force to some degree, but we found that a magnet with a length/diameter ratio of 2.0 is optimal, as no significant force increase is achieved by longer magnets (data not shown). Additionally, a short distal magnet facilitates the design of both simple straight and complex custom-designed probes.

The magnetic attraction force was measured using a tensiometer (Model 5564, Intron Ltd., Buckinghamshire, UK). Details on this measurement system have been reported previously [7, 8]. Briefly, the ex vivo bowel segment is fixed onto a support board with a free $15.0 \mathrm{~cm}$ segment (Figure 2(c)) and placed in a water bath at $37^{\circ} \mathrm{C}$. After injection magnetisation, a magnetic probe connected to the tensiometer load cell was brought into contact with the bowel for retraction force measurement. Figure 2(d) illustrates a recorded retraction force-distance curve.

Several parameters can be derived from the recorded retraction force and distance curve (Figure 2(d)). Peak force $\left[F_{\max }(\mathrm{N})\right]$ represents the maximal attraction (or bonding) force between the magnet and the magnetised tissue, and after reaching $F_{\max }$, the probe starts to separate from the bowel until it detaches completely from it. The stress $[\sigma(\mathrm{Pa})]$ at peak force is derived from dividing $F_{\max }$ by the probe endsurface area. Work $[W(\mathrm{~J})]$ is defined as the area under the force-distance curve, which represents the work required for detachment of the two systems [23]. Work is calculated by 
TABLE 1: Characteristics of the magnetic particles.

\begin{tabular}{|c|c|c|c|c|}
\hline & Size (diameter) & $M_{s}(\mathrm{emu} / \mathrm{g})$ & $H_{c}(\mathrm{kOe})$ & $\mathrm{Fe} / \mathrm{Cr}(\mathrm{w} \%)$ \\
\hline SS410- $\mu$ Ps & Up to $50 \mu \mathrm{m}^{\text {SEM }}$ & 168.0 & 0.01 & $86.6 \pm 6.8 / 12.6 \pm 1.0$ \\
\hline SS $430-\mu \mathrm{Ps}$ & Up to $40 \mu \mathrm{m}^{\mathrm{SEM}}$ & 110.0 & 0.02 & $82.2 \pm 6.7 / 16.9 \pm 1.4$ \\
\hline
\end{tabular}

Magnetisation at saturation $\left(M_{s}\right)$ and coercivity $\left(H_{c}\right)$ measured with a SQUID magnetometer at $300 \mathrm{~K}$; iron and chromium percentage as deduced from ICPOES measurements and size as obtained by SEM characterizations.

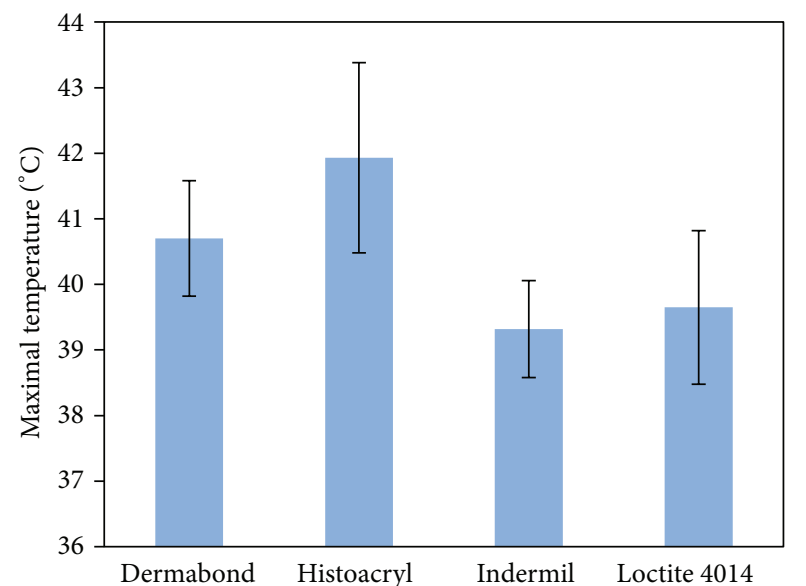

FIGURE 3: Averaged maximum temperatures were measured by the IR thermal camera after $0.5 \mathrm{~mL}$ of each glue was deposited onto the surface of ex vivo porcine bowel (error bar: standard deviation, number of test $n=7$ ).

a custom-written MATLAB (MathWorks, Cambridge, UK) program using (1) which is based on linear trapezoidal rule [24]:

$$
W=\sum_{i=0}^{N-1}\left(x_{i+1}-x_{i}\right) * \frac{\left(F_{i}+F_{i+1}\right)}{2}
$$

where $W$ is adhesion work, $x$ is retraction distance, $F$ is retraction force, $i$ is data sampling point, and $N$ is total number of data points.

\section{Results}

3.1. Heat Generation by Glues. The maximal temperature generated during glue polymerisation by the 4 medical grade cyanoacrylate glues studied was obtained from the recorded thermal camera image. Figure 3 plots the averaged maximal temperatures measured by the IR thermal camera after $0.5 \mathrm{~mL}$ of each glue was deposited onto the surface of ex vivo porcine bowel (error bar: standard deviation, number of test $n=7$ ). All showed safe heat generation (i.e., below $42^{\circ} \mathrm{C}$ ) except for Histoacryl (maximal about $43.5^{\circ} \mathrm{C}$ ).

3.2. Characterisation of Magnetic Particles. Figures 4(a) and 4(b) present the appearance of stainless steel microparticles as observed by electron microscopy and Figure 4(c) the hysteresis curves at room temperature.

Results from XRD (not presented) and electron microscopy findings showed both SS410 and SS430 particles
TABLE 2: Magnetic bowel retraction data.

\begin{tabular}{lccc}
\hline & $F_{\max }(\mathrm{N})$ & $W(\mathrm{~mJ})$ & $\sigma(\mathrm{kPa})$ \\
\hline $5 \mathrm{~mm}$ magnet probe & $2.2 \pm 0.2$ & $34 \pm 11$ & $114 \pm 11.7$ \\
$10 \mathrm{~mm}$ magnet probe & $5.1 \pm 0.3$ & $81 \pm 17$ & $65 \pm 4.3$ \\
\hline
\end{tabular}

SS410- $\mu$ Ps-based magnetic glues at concentration $1 \mathrm{~g} / \mathrm{mL}$ : intraluminally injected volume of $2 \mathrm{~mL}$.

to be crystalline but polydispersed in size and shape with particles' diameters ranging from a few microns up to $50 \mu \mathrm{m}$ (Figures 4(a) and 4(b)). Magnetic properties in terms of magnetization as a function of applied magnetic field were quantified at room temperature and the resulting curves are shown in Figure 4(c). As expected the curves are symmetric and the magnetization at saturation, $M_{s}$, is deduced from the plateau, when the magnetization does not increase any further with increasing magnetic field strength. SS410- $\mu$ Ps (Figure 4(c1)) exhibit a higher magnetization at saturation than SS430- $\mu$ Ps, and this is likely due to their slightly higher iron content and a lower chromium doping (Table 1). The coercivity refers to the magnetic field which needs to be applied to reduce the magnetization of a material down to zero after the magnetization of the sample has reached saturation. For both SS- $\mu$ Ps the remanence (i.e., the remaining magnetisation after the field has been removed) has been found to be very small. These observations indicate that both stainless steel $\mu$ Ps even though too large to form stable suspension would not present difficulty in dispersion in the fluid due to magnetic interactions since the particles exhibit no mutual magnetic attraction unless placed in a magnetic field. SS410- $\mu$ Ps were chosen for the study in view of their higher magnetization and lower coercivity (or remanence).

\subsection{Measurement of Magnetic Bowel Retraction and Forces.} Harvested porcine colonic segments were used with injection by a large gauge needle (16 G or $1.7 \mathrm{~mm}$ diameter $\times 50 \mathrm{~mm}$, B. Braun Melsungen AG) to facilitate rapid injection of high concentration magnetic glue, although a smaller gauge needle (19G needle from BD Microlance 3, BD Drogheda, Ireland) can also be used for slower injection. After withdrawal of the needle, the small puncture wound effectively sealed itself by the injected glue with no visible leakage. The procedure took around 1 minute. Table 2 summarises the magnetic retraction of a magnetised bowel segments in 20 ex vivo experiments using both the $5 \mathrm{~mm}$ and the $10 \mathrm{~mm}$ magnet probes.

The average pull force that surgeons use to provide sufficient tension to the bowel is $2.5 \mathrm{~N}$ [1] with the maximal force being just below $5 \mathrm{~N}$. The test results obtained in the present 

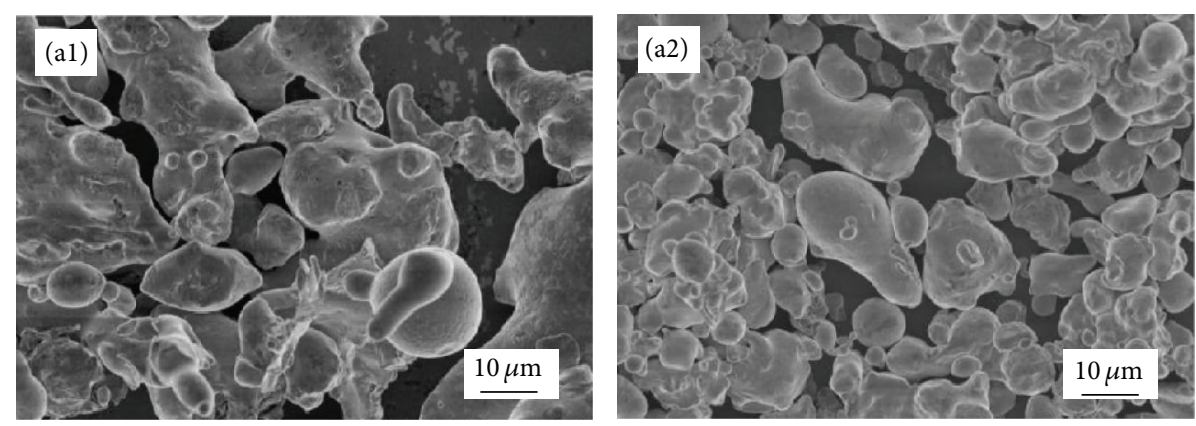

(a)
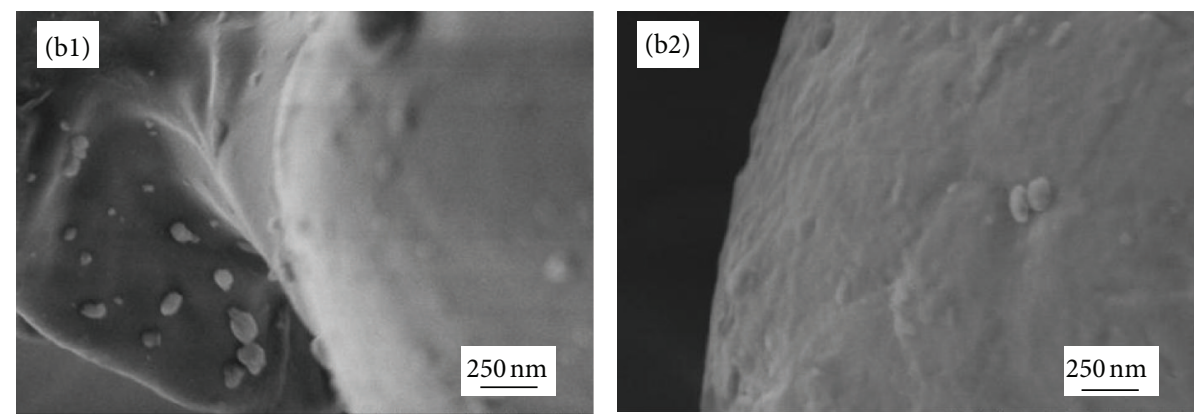

(b)

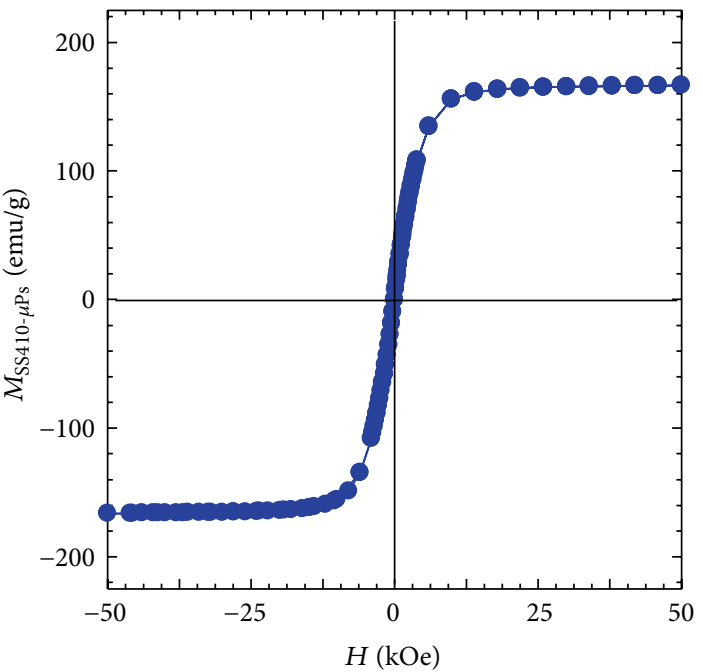

$(\mathrm{c} 1)$

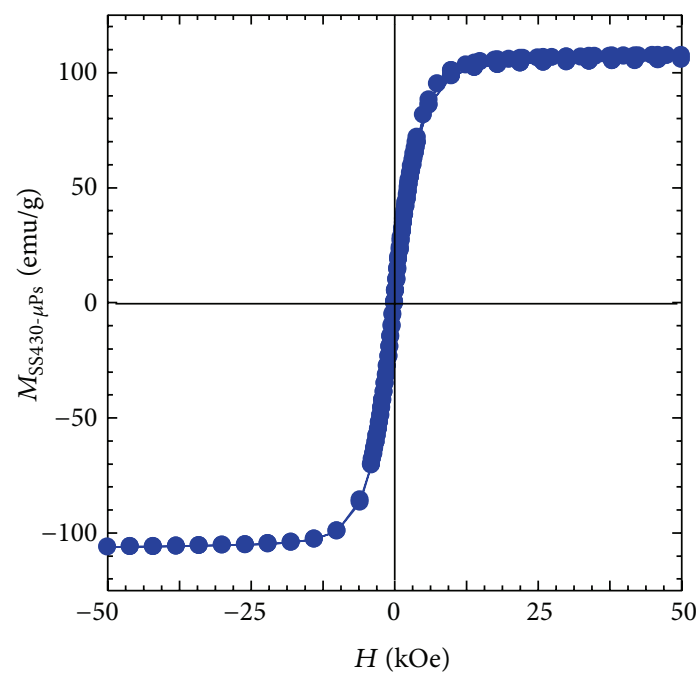

$(\mathrm{c} 2)$

(c)

FIGURE 4: (a)-(b) SEM images and (c) hysteresis curves completed at room temperature for two stainless steel microparticle of SS410- $\mu$ Ps (1) and SS430- $\mu$ Ps (2).

study (Table 2) indicate that SS410- $\mu$ Ps based bowel magnetization is capable of providing retraction forces and work in this range with use of 5 and $10 \mathrm{~mm}$ diameter permanent magnet probes. One important advantage of magnetic retraction over conventional pull traction by teeth-like graspers is that it inflicts less stress during high tension retraction with the tissues being not only retracted but compressed between the ridges of the graspers. For example, average pressures at the tip of conventional graspers at the contact surface area with the target tissue vary from $210 \mathrm{kPa}$ to $650 \mathrm{kPa}$ [25]. In contrast, the current approach does not require such compression and much lower pressures (65 $\mathrm{kPa}$ to $114 \mathrm{kPa}$ ) were observed in the current experiments by the $10 \mathrm{~mm}$ and $5 \mathrm{~mm}$ diameter magnetic probes, respectively (Table 2 ).

3.4. On-Going Iron Oxide Nanoparticles Formulations. Magnetic nanoparticles can potentially exhibit stronger magnetic properties than microparticles per unit mass. This arises because bulk magnetic materials present multiple domains of magnetisations. Within each domain, the magnetisation has only one direction, which however can vary from one domain to another. The presence of several interacting domains in 
one particle with potentially different orientations can result in lowering the overall magnetisation of the particle. In contrast, decrease of the size of the particles to a single domain excludes the possibility of magnetic interactions between multiple magnetic domains within a single particle. If the nanoparticles do not carry a magnetic "dead-layer" at their interface, their magnetic properties can be enhanced compared to microparticles when exposed to the same magnetic field [26-28].

Magnetic nanoparticles have been successfully used for numerous biomedical applications [29-34]; however we found that iron oxide nPs could not be mixed easily with Loctite 4014 liquid for medical magnetic glue injection. For this reason the on-going studies by the group is exploring ways to overcome this problem as it is related to the fast polymerization of the glue when mixed with the iron oxide nanoparticles. This is likely associated with the surface reactivity of the nanoparticles which needs to be adequately tuned and possibly coated to enable development of efficient nanoparticle-based medical magneto-glues.

\section{Discussion}

The new system based on formation of intraluminal magnetic coagulum provides highly effective atraumatic retraction and overcomes the problems reported with previous approaches based on localised ferromagnetisation of tissues, that is, low injection volume during interstitial ferromagnetisation [8] and peeling of surface magnetic pellets beyond certain retraction forces [7]. Furthermore, it provides significantly greater retraction forces, which meet all the requirements for uncompromised bowel retraction/manipulation during MAS equivalent to bowel grasping without risk of trauma or slippage. Additionally, the results of the present experiments have excluded thermal injury to the issues induced by medical grade cyanoacrylate glues during polymerisation. Loctite 4014 glue was selected because of its very low viscosity, single component nature, rapid cure rate, and ability to mix with stainless steel microparticles.

To date, ex vivo porcine bowel segments have been used to validate the concept and measure the forces generated (Figure 5). To facilitate intraluminal injection at randomly selected target bowel segment, an open abdominal model was used instead of a laparoscopic model. Two $\mathrm{mL}$ magnetic glue was injected intraluminally into targeted bowel segments and the $10 \mathrm{~mm}$ diameter magnet probe used to retract the magnetised bowel (Figure 5(a)) which could be manipulated and moved around, and retracted (Figure 5(b)) with maximal pull detachment force of $5 \mathrm{~N}$, which was in agreement with Instron bench test results using ex vivo bowel segments (Table 2).

The ex vivo experiments with the formulated magnetic glue also demonstrated that even small volumes $(2 \mathrm{~mL})$ injected intraluminally in target bowel segments enable effective magnetic bowel retraction using a small $(5$ to $10 \mathrm{~mm}$ diameter) permanent magnet probes. For the same retraction force, the experimental data confirmed that the stress exerted on the target tissue by magnetic probes was significantly less when compared with the force generated during retraction

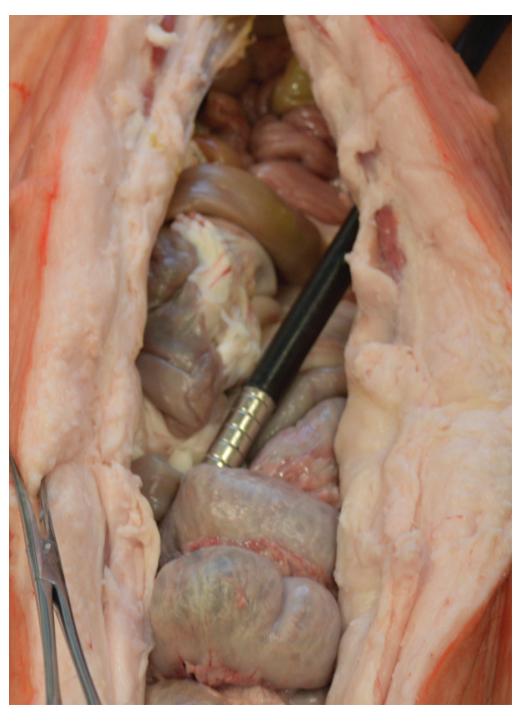

(a)

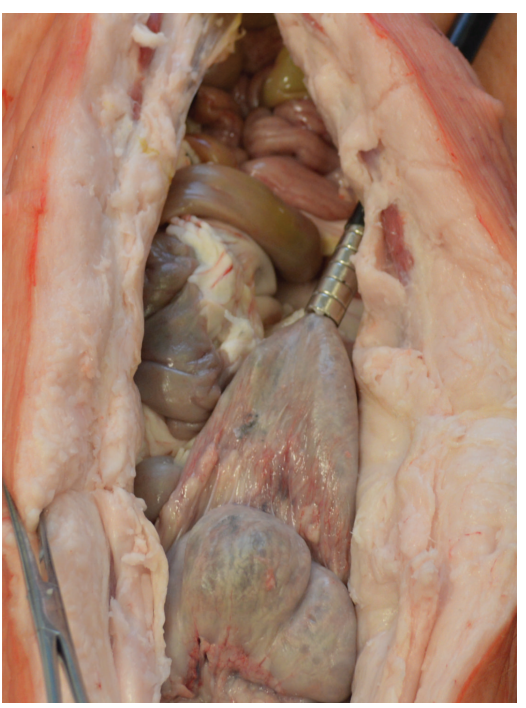

(b)

Figure 5: Retraction of in situ magnetised bowel using an ex vivo porcine model and a volume of $2 \mathrm{~mL}$ magnetic media with concentration of $1 \mathrm{~g} / \mathrm{mL}$ was injected into a bowel segment: (a) photography showing the $10 \mathrm{~mm}$ magnet probe inserted into a $10 \mathrm{~mm}$ port and engaged with the magnetised bowel segment; (b) photography showing retraction of the magnetised bowel toward the port direction, with maximal detachment force of $5 \mathrm{~N}$.

by grasping forceps ( $65 \mathrm{kPa}$ versus $210 \mathrm{kPa}$ ). Furthermore, the magnetic probe does not gather the tissue into a fold but instead creates a flat and smooth contact area with a wider distribution of probe-tissue contact forces. This underlies the atraumatic nature of magnetic retraction since the tissue falls off when the force exceeds the magnetic pull. Active release of the magnetically retracted tissue can be obtained by custom-designed probes which adjust the magnetic attraction force through a controlled release mechanism.

Our experiments also confirmed that change of retraction speed (from $0.1 \mathrm{~mm} / \mathrm{s}$ to $10 \mathrm{~mm} / \mathrm{s}$ ) does not affect the 
magnetic retraction force and work, suggesting that bowel retracted with such probes would not have to be moved slowly during surgery. The experiments demonstrate that compared to external (serosal) application, the intraluminal magnetic coagulum significantly increases the maximal retraction force $(1.75 \pm 0.86 \mathrm{~N}$ versus $5.15 \pm 0.98 \mathrm{~N})$ with the use of a $10 \mathrm{~mm}$ magnet probe. Once the external magnet probe is removed, the coagulum detaches from the mucosa and thus becomes susceptible to spontaneous elimination in the stools. An adjustable magnetic force probe is desirable for controlled release of tissue whenever this is required. A simple and effective design for such a probe has been designed and developed in our lab and will be published elsewhere.

\section{Conclusions}

The results of the current experimental study confirm that, with the technology described, intraluminal magnetic coagula formed from medical-grade biocompatible cyanoacrylate (Loctite) and SS410- $\mu$ Ps constitutes a novel system for efficient and atraumatic magnetic retraction of bowel. The system provides the retraction forces required for bowel manipulation and handling during laparoscopic surgery. The technology functions by producing a coagulum containing a sufficient mass of aggregated stainless steel microparticles adherent to the mucosa. This is either removed with the specimen in resectional bowel surgery or is expelled with return of bowel function. Further improvement is foreseen with the use of polymer coated nanoparticles of iron oxide being developed in our laboratory instead of stainless steel microparticles. Evaluation of the fully developed technology by in vivo large animal studies before translation to clinical practice is needed.

\section{Conflict of Interests}

The authors declare that there is no conflict of interests regarding the publication of this paper.

\section{Acknowledgment}

This work was supported by the Engineering and Physical Sciences Research Council (EPSRC), UK, under Grant EP/HO $10033 / 1$.

\section{References}

[1] E. A. M. Heijnsdijk, H. de Visser, J. Dankelman, and D. J. Gouma, "Slip and damage properties of jaws of laparoscopic graspers," Surgical Endoscopy and Other Interventional Techniques, vol. 18, no. 6, pp. 974-979, 2004.

[2] E. A. M. Heijnsdijk, J. Dankelman, and D. J. Gouma, "Effectiveness of grasping and duration of clamping using laparoscopic graspers," Surgical Endoscopy and Other Interventional Techniques, vol. 16, no. 9, pp. 1329-1331, 2002.

[3] P.-O. Park, M. Bergström, K. Ikeda, A. Fritscher-Ravens, and P. Swain, "Experimental studies of transgastric gallbladder surgery: cholecystectomy and cholecystogastric anastomosis (videos)," Gastrointestinal Endoscopy, vol. 61, no. 4, pp. 601-606, 2005.

[4] M. Ryou and C. C. Thompson, "Magnetic retraction in naturalorifice transluminal endoscopic surgery (NOTES): addressing the problem of traction and countertraction," Endoscopy, vol. 41, no. 2, pp. 143-148, 2009.

[5] Q. A. Pankhurst, J. Connolly, S. K. Jones, and J. Dobson, "Applications of magnetic nanoparticles in biomedicine," Journal of Physics D, vol. 36, no. 13, pp. R167-R181, 2003.

[6] R. A. Frimpong and J. Z. Hilt, "Magnetic nanoparticles in biomedicine: synthesis, functionalization and applications," Nanomedicine, vol. 5, no. 9, pp. 1401-1414, 2010.

[7] Z. Wang, L. Wang, B. Tang, T. Frank, S. Brown, and A. Cuschieri, "Retraction by surface ferromagnetisation of target tissues: preliminary studies on feasibility of magnetic retraction for endoscopic surgery," Surgical Endoscopy and Other Interventional Techniques, vol. 22, no. 8, pp. 1838-1844, 2008.

[8] Z. Wang, L. Wang, S. I. Brown, T. G. Frank, and A. Cuschieri, "Ferromagnetization of target tissues by interstitial injection of ferrofluid: formulation and evidence of efficacy for magnetic retraction," IEEE Transactions on Biomedical Engineering, vol. 56, no. 9, pp. 2244-2252, 2009.

[9] G. G. M. Pinkse, E. Steenvoorde, S. Hogendoorn et al., "Stable transplantation results of magnetically retracted islets: a novel method," Diabetologia, vol. 47, no. 1, pp. 55-61, 2004.

[10] D. C. Meeker, E. H. Maslen, R. C. Ritter, and F. M. Creighton, "Optimal realization of arbitrary forces in a magnetic stereotaxis system," IEEE Transactions on Magnetics, vol. 32, no. 2, pp. 320328, 1996.

[11] S. Martel, J.-B. Mathieu, O. Felfoul et al., "Automatic navigation of an untethered device in the artery of a living animal using a conventional clinical magnetic resonance imaging system," Applied Physics Letters, vol. 90, no. 11, Article ID 114105, 2007.

[12] J. Cadeddu, R. Fernandez, M. Desai et al., "Novel magnetically guided intra-abdominal camera to facilitate laparoendoscopic single-site surgery: initial human experience," Surgical Endoscopy and Other Interventional Techniques, vol. 23, no. 8, pp. 1894-1899, 2009.

[13] M. T. Gettman and P. Swain, "Initial experimental evaluation of wireless capsule endoscopes in the bladder: implications for capsule cystoscopy," European Urology, vol. 55, no. 5, pp. 1207$1212,2009$.

[14] T. Uchiyama, K. Mohri, M. Shinkai et al., "Position sensing of magnetite gel using MI sensor for brain tumor detection," IEEE Transactions on Magnetics, vol. 33, no. 5, pp. 4266-4268, 1997.

[15] J. M. Peeters, J.-H. Seppenwoolde, L. W. Bartels, and C. J. G. Bakker, "Development and testing of passive tracking markers for different field strengths and tracking speeds," Physics in Medicine and Biology, vol. 51, no. 6, pp. N127-N137, 2006.

[16] T. Ohdaira and H. Nagai, "Intraoperative localization of earlystage upper gastrointestinal tumors using a magnetic marking clip-detecting system," Surgical Endoscopy and other Interventional Techniques, vol. 21, no. 5, pp. 810-815, 2007.

[17] D. Erdmann, R. Sweis, C. Heitmann et al., "Side-to-side sutureless vascular anastomosis with magnets," Journal of Vascular Surgery, vol. 40, no. 3, pp. 505-511, 2004.

[18] N. Muraoka, H. Uematsu, E. Yamanouchi et al., "Yamanouchi magnetic compression anastomosis for bilioenteric anastomotic stricture after living-donor liver transplantation," Journal of Vascular and Interventional Radiology, vol. 16, no. 9, pp. 12631267, 2005. 
[19] A. J. Singer, T. Zimmerman, J. Rooney, P. Cameau, G. Rudomen, and S. A. McClain, "Comparison of wound-bursting strengths and surface characteristics of FDA-approved tissue adhesives for skin closure," Journal of Adhesion Science and Technology, vol. 18, no. 1, pp. 19-27, 2004.

[20] M. A. Astrahan, M. D. Sapozink, D. Cohen et al., "Microwave applicator for transurethral hyperthermia of benign prostatic hyperplasia," International Journal of Hyperthermia, vol. 5, no. 3, pp. 283-296, 1989.

[21] H. Chen, M. D. Kaminski, A. D. Ebner, J. A. Ritter, and A. J. Rosengart, "Magnetizable intravascular stents for sequestration of systemically circulating magnetic nano- and microspheres," in Proceedings of the 3rd IEEE/EMBS Special Topic Conference on Microtechnology in Medicine and Biology, pp. 286-289, May 2005.

[22] H. Chen, A. D. Ebner, D. Bockenfeld et al., "A comprehensive in vitro investigation of a portable magnetic separator device for human blood detoxification," Physics in Medicine and Biology, vol. 52, no. 19, pp. 6053-6072, 2007.

[23] C. Eouani, P. Piccerelle, P. Prinderre, E. Bourret, and J. Joachim, "In-vitro comparative study of buccal mucoadhesive performance of different polymeric films," European Journal of Pharmaceutics and Biopharmaceutics, vol. 52, no. 1, pp. 45-55, 2001.

[24] Pharmacokinetics, Marcel Dekker, New York, NY, USA, 1982, edited by M. Gibaldi and M. Perrier.

[25] J. A. Cartmill, A. J. Shakeshaft, W. R. Walsht, and C. J. Martin, "High pressures are generated at the tip of laparoscopic graspers," Australian and New Zealand Journal of Surgery, vol. 69, no. 2, pp. 127-130, 1999.

[26] M. Mikhaylova, D. K. Kim, N. Bobrysheva et al., "Superparamagnetism of magnetite nanoparticles: dependence on surface modification," Langmuir, vol. 20, no. 6, pp. 2472-2477, 2004.

[27] C. d. Montferrand, Y. Lalatonne, D. Bonnin et al., "Sizedependent nonlinear weak-field magnetic behavior of maghemite nanoparticles," Small, vol. 8, no. 12, pp. 19451956, 2012.

[28] U. Jeong, X. Teng, Y. Wang, H. Yang, and Y. Xia, "Superparamagnetic colloids: controlled synthesis and niche applications," Advanced Materials, vol. 19, no. 1, pp. 33-60, 2007.

[29] G. A. O. Jinhao, G. U. Hongwei, and X. U. Bing, "Multifunctional magnetic nanoparticles: design, synthesis, and biomedical applications," Accounts of Chemical Research, vol. 42, no. 8, pp. 1097-1107, 2009.

[30] M. Oudkerk, A. G. van den Heuvel, P. A. Wielopolski, P. I. M. Schmitz, I. H. M. Borel Rinkes, and T. Wiggers, "Hepatic lesions: detection with ferumoxide-enhanced T1-weighted MR imaging," Radiology, vol. 203, no. 2, pp. 449-456, 1997.

[31] R. C. Semelka and T. K. G. Helmberger, "State of the art: contrast agents for mr imaging of the liver," Radiology, vol. 218, no. 1, pp. 27-38, 2001.

[32] S. Chen, C. Hoskins, L. Wang, M. P. MacDonald, and P. André, "A water-soluble temperature nanoprobe based on a multimodal magnetic-luminescent nanocolloid," Chemical Communications, vol. 48, no. 19, pp. 2501-2503, 2012.

[33] S. Chen, L. Wang, S. L. Duce et al., "Engineered biocompatible nanoparticles for in vivo imaging applications," Journal of the American Chemical Society, vol. 132, no. 42, pp. 15022-15029, 2010.

[34] L. Motte, F. Benyettou, C. de Beaucorps, M. Lecouvey, I. Milesovic, and Y. Lalatonne, "Multimodal superparamagnetic nanoplatform for clinical applications: immunoassays, imaging \& therapy," Faraday Discussions, vol. 149, pp. 211-225, 2011. 

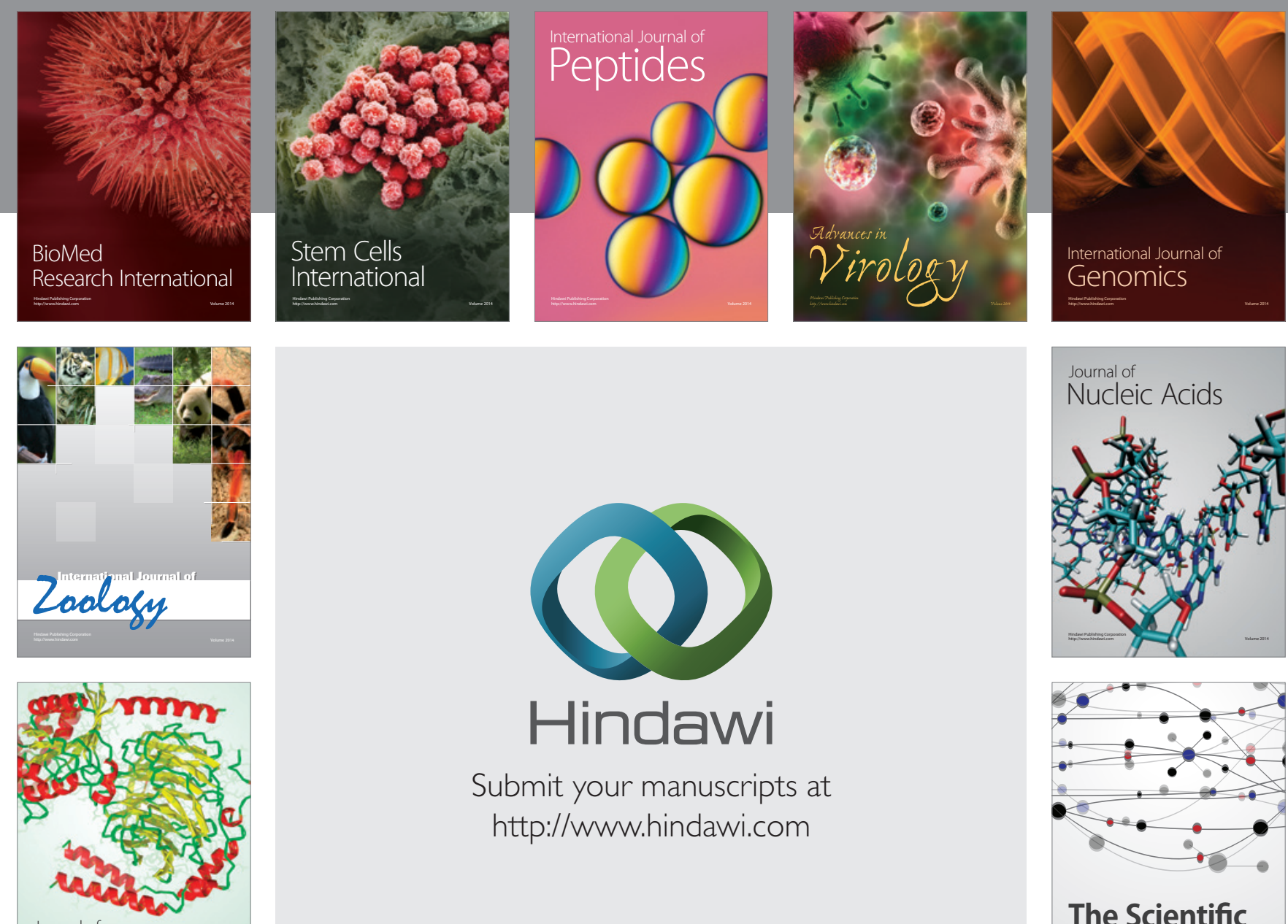

Submit your manuscripts at

http://www.hindawi.com

Journal of
Signal Transduction
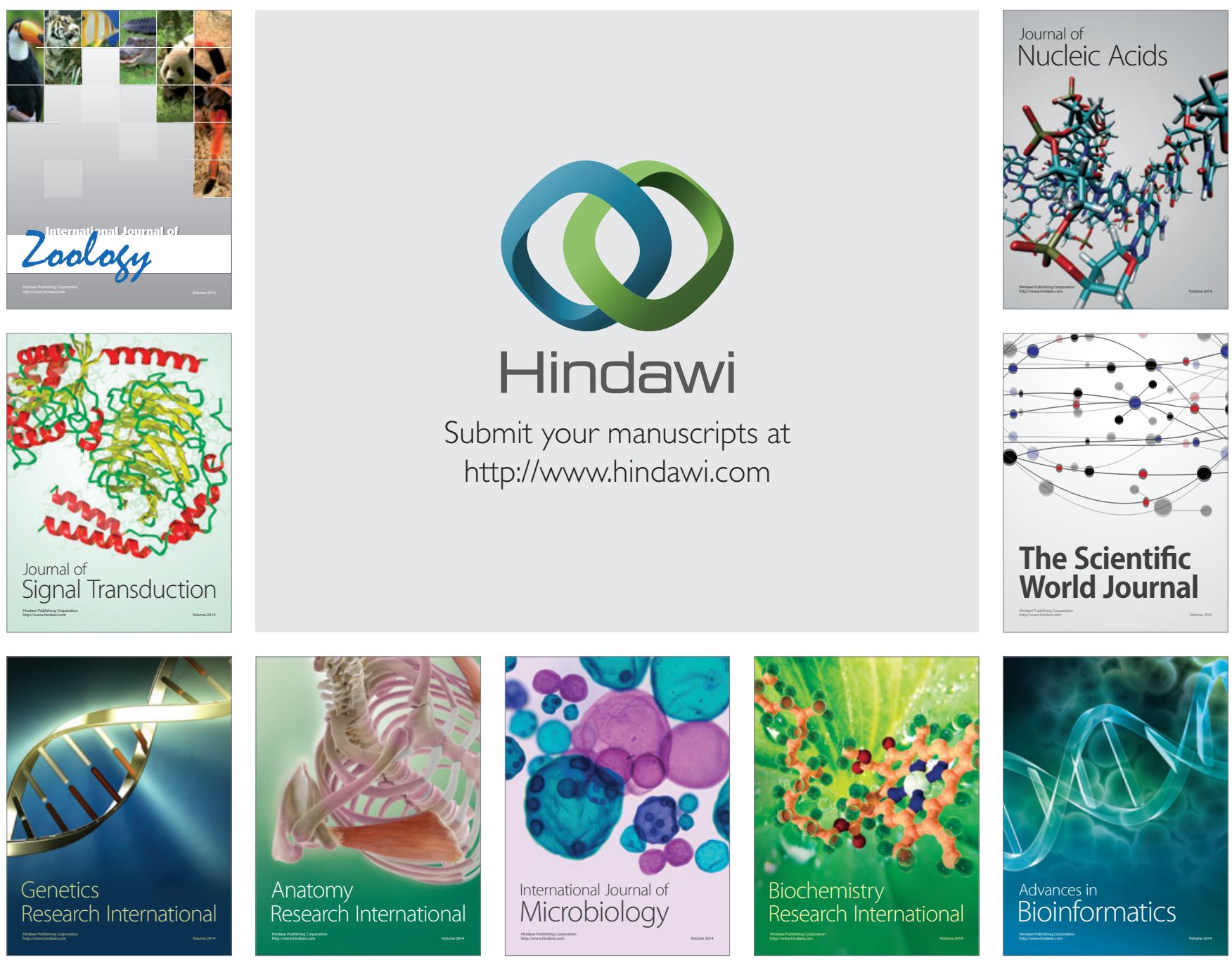

The Scientific World Journal
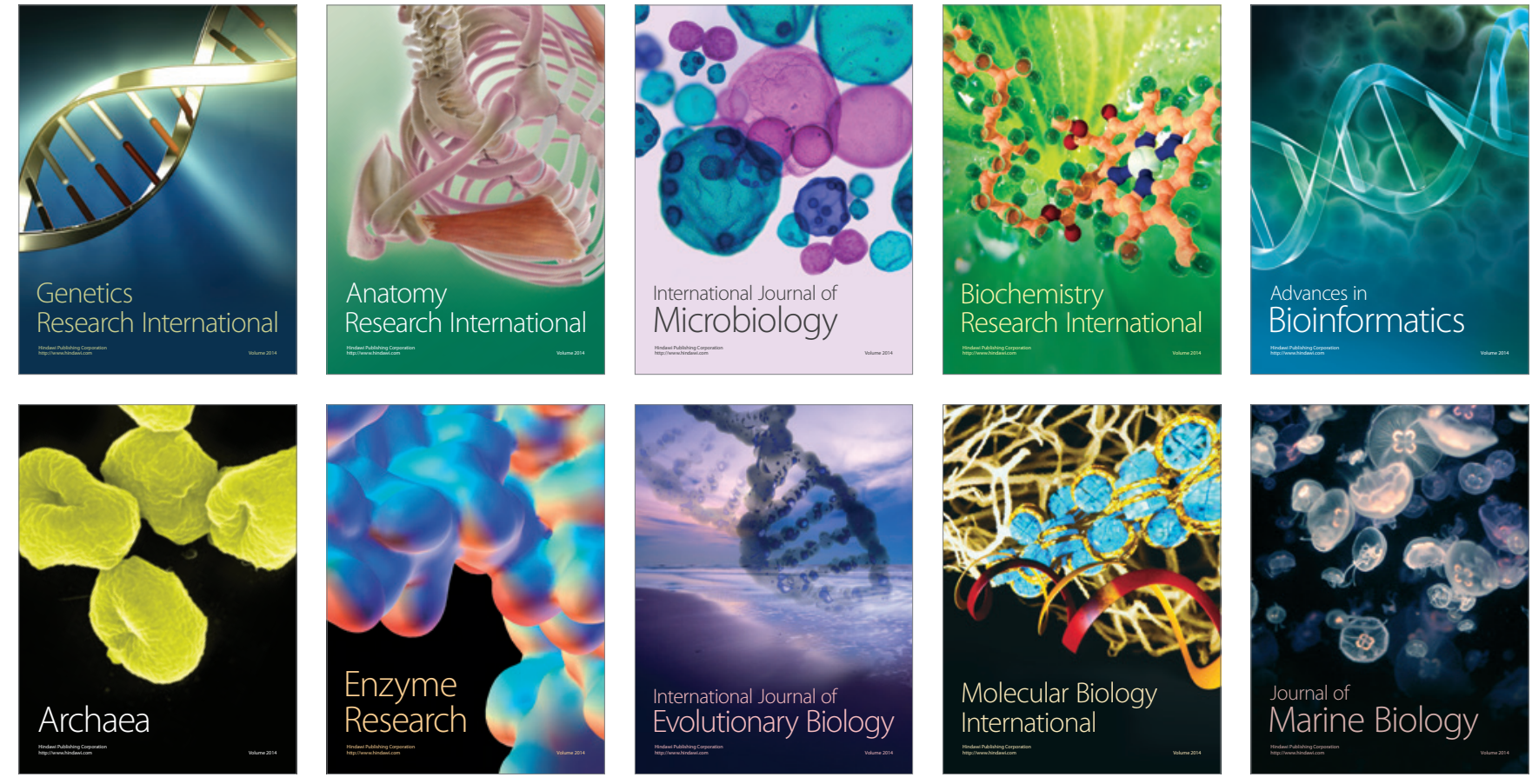